\title{
Delayed-onset white cord syndrome after anterior and posterior cervical decompression surgery for symptomatic ossification of spinal ligaments: illustrative cases
}

\author{
Ranjit D. Singh, MD, MSc, Mark P. Arts, MD, PhD, and Godard C. W. de Ruiter, MD, PhD \\ Department of Neurosurgery, Haaglanden Medical Center, University Neurosurgical Center Holland, The Hague, The Netherlands
}

BACKGROUND White cord syndrome is an extremely rare complication of cervical decompressive surgery, characterized by serious postoperative neurological deficits in the absence of apparent surgical complications. It is named after the characteristic ischemic-edematous intramedullary T2hyperintense signal on postoperative magnetic resonance imaging and is believed to be caused by ischemic-reperfusion injury. Neurological deficits typically manifest immediately after surgery, and delayed occurrence has been reported only once.

OBSERVATIONS The authors presented two cases of delayed white cord syndrome after anterior and posterior cervical decompression surgery for symptomatic ossification of the posterior longitudinal ligament and ligamentum flavum, respectively. Neurological deficits manifested on postoperative day 2 (case 1) and day 8 (case 2). The patients' conditions were managed with high-dose corticosteroids, mean arterial pressure augmentation, and early physical therapy, after which they showed partial neurological recovery at discharge, which improved further by the 3-month follow-up visit.

LESSONS The authors' aim was to raise awareness among spine surgeons about this rare but severe complication of cervical decompressive surgery and to emphasize the mainstays of treatment based on current best evidence: high-dose corticosteroids, mean arterial pressure augmentation, and early physical therapy.

https://thejns.org/doi/abs/10.3171/CASE2113

KEYWORDS white cord syndrome; ischemic-reperfusion spinal cord injury; cervical ossification of the posterior longitudinal ligament; OPLL; cervical ossification of the ligamentum flavum; OLF

Ossification of spinal ligaments is a potential cause of cervical radiculomyelopathy and is mainly, but not exclusively, seen in East Asian populations, particularly Japanese individuals. ${ }^{1}$ Ossification of the posterior longitudinal ligament (OPLL) occurs most commonly in the cervical spine, with an incidence of approximately $6 \%$ in the Japanese population. ${ }^{2}$ Conversely, ossification of the ligamentum flavum (OLF) in the cervical spine is extremely rare, with a relative incidence of $<1 \%{ }^{3}$ For symptomatic cases, cervical decompressive procedures such as anterior corpectomy with placement of a cage, anterior cervical discectomy and fusion, and posterior cervical laminectomy with or without instrumented fusion are frequently performed and are often successful in halting progression or improving symptoms. The exact surgical approach typically depends on case-specific anatomical factors, such as the site of spinal cord compression and the patient's age and general health. Postoperative neurological deficits seldom occur and are mostly attributable to iatrogenic injury or postoperative hematoma. ${ }^{4}$
An extremely rare but severe complication of cervical decompression surgery is "white cord syndrome," named after the characteristic T2-hyperintense intramedullary signal on postoperative magnetic resonance imaging (MRI) that represents spinal cord ischemia and/or edema. It was first described by Chin and colleagues in 2013; to the best of our knowledge, only a few reports have been published since..$^{5-12}$ Clinically, it is characterized by severe neurological deficits such as hemi- or quadriplegia with intraoperative (measured by neuromonitoring) or direct postoperative onset, although a delayed manifestation also has been described. ${ }^{8}$ The prevailing hypothesis assumes that the syndrome is caused by reperfusion injury after sudden decompression of a chronically compressed spinal cord segment, resulting in acutely increased blood supply to the affected area, which leads to subsequent damage by either direct trauma from blood or oxygenfree radicals within the newly available blood. We describe two cases of white cord syndrome with delayed onset after both

ABBREVIATIONS CT = computed tomography; MAP = mean arterial pressure; MRI = magnetic resonance imaging; OLF = ossification of the ligamentum flavum; OPLL $=$ ossification of the posterior longitudinal ligament.

INCLUDE WHEN CITING Published May 10, 2021; DOI: 10.3171/CASE2113.

SUBMITTED January 5, 2021. ACCEPTED February 10, 2021.

(C) 2021 The authors, CC BY-NC-ND 4.0 (http://creativecommons.org/licenses/by-nc-nd/4.0/). 

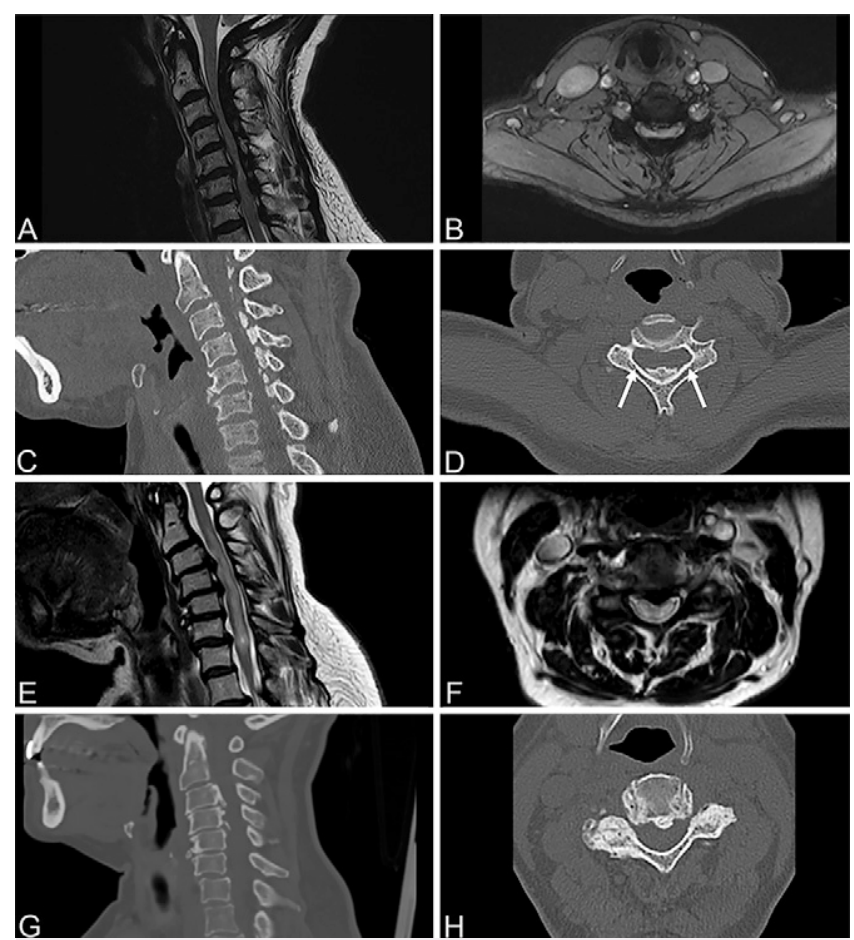

FIG. 1. A-D: Preoperative cervical T2-weighted MRI of case 1 showing spinal stenosis at levels $\mathrm{C} 3-6$ with diffuse thickening of the ligamentum flavum and a weak T2-hyperintense signal at levels C3-4 and C5-6. Sagittal (A) and axial (B) images show level C5-6. Preoperative sagittal (C) and axial (D) CT scans demonstrate multilevel OLF, most prominent on level C5-6. White arrows illustrate the location of the milled slots using the Scampi technique for cervical decompression (D). E-H: Preoperative cervical T2-weighted MRI of case 2 showing spinal stenosis at levels C3-4 and C4-5 with an elongated T2-hyperintense signal ranging from $\mathrm{C} 2-3$ to $\mathbf{C 6}-7$. Sagittal $(\mathbf{E})$ and axial $(\mathbf{F})$ images show level C3-4. Preoperative CT scans demonstrate OPLL at levels C3-4 and C4-5 and a slight swan-neck deformity. Sagittal (G) and axial (H) images show level C3-4.

anterior and posterior decompressive spinal surgery performed for symptomatic cervical OPLL and OLF, respectively.

\section{Illustrative Cases}

\section{Case 1}

Presentation

A 59-year-old Dutch woman of African descent presented with progressive neck pain, tingling sensations in both legs without dermatomal distribution, and a spastic gait for the past 2 months. The patient also mentioned occasional involuntary defecation during micturition and decreased perianal sensation that had existed for the past several months but had not worsened lately. Her medical history indicated hypertension, diabetes mellitus type II, and a parathyroidectomy performed almost 10 years earlier because of primary hyperparathyroidism. Neurological examination on admission found no clear loss of muscle strength despite some antalgic restrictions, a spotty pattern of hypesthesia in the left leg, and similarly distributed allodynia in the right leg. Preoperative MRI demonstrated significant spinal stenosis ranging from C3 to C6, with spinal cord compression caused by diffuse thickening of the ligamentum flavum with suspected ossification and weak T2-hyperintense signal on levels $\mathrm{C} 3-4$ and $\mathrm{C} 5-6$ (Fig. $1 \mathrm{~A}$ and $\mathrm{B}$ ). Computed tomography (CT) imaging confirmed the diagnosis of multilevel cervical OLF, with level C5-6 being most severely affected (Fig. 1C and D). The patient underwent an elective posterior decompressive laminectomy from C3 to C6.

\section{Operation}

After the patient was placed prone and the paravertebral muscles were mobilized, the spinous processes of C3-6 were removed with a Leksell rongeur. Next, two slots were milled in the laminae of C3-6 on the medial side of the facet joints. Our intention was to subsequently remove the vertebral arches together with the ossified ligament in one piece starting from the caudal side using the "Scampi" technique while avoiding iatrogenic central spinal cord compression (Fig. 2). However, because of superior and inferior extension of ossifications continuous with the laminae of both $\mathrm{C} 2$ and $\mathrm{C} 7$, this maneuver was not directly possible. The ossified ligament was therefore cut between the laminae of $\mathrm{C} 6$ and $\mathrm{C} 7$ using a Kerrison rongeur. As a result of tight adherence of the ossified ligamentum flavum to the dura mater, cranial elevation of the vertebral arches was still not possible without risking a major dural defect. Therefore, the dura mater was first carefully dissected away from the ossified ligamentum

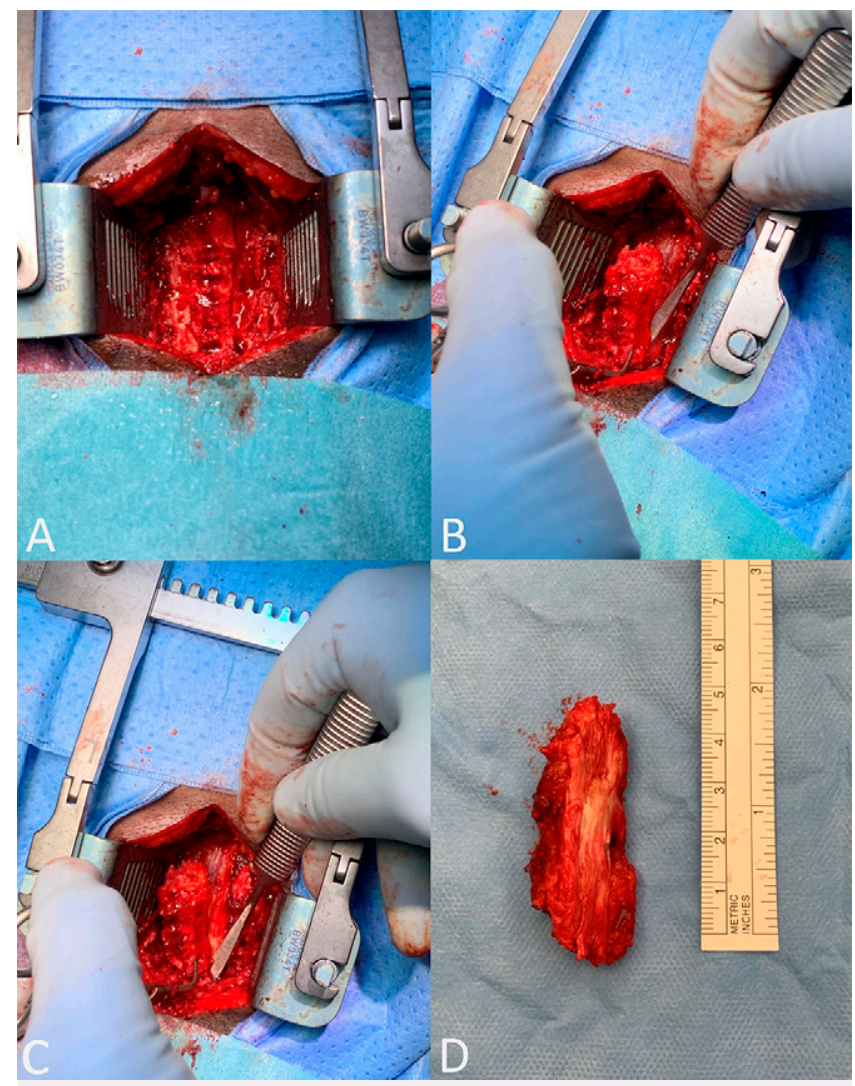

FIG. 2. Intraoperative view of bilateral slots in lamina (A), dissection of dura mater from ossified ligament (B), caudocranial elevation of vertebral arches $(\mathbf{C})$, and postoperative en bloc resected specimen (D). 


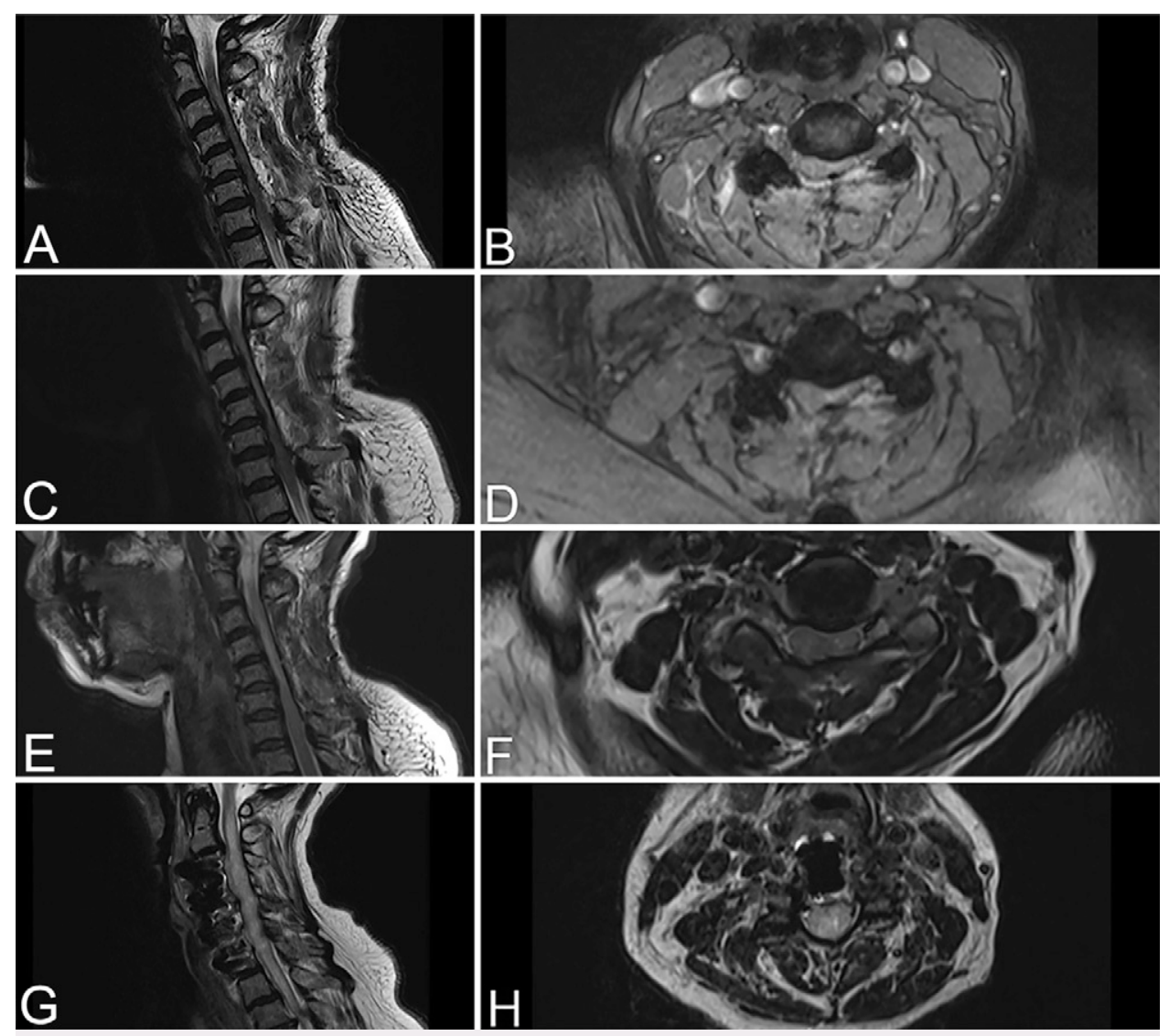

FIG. 3. Postoperative cervical T2-weighted sagittal and axial MRI scans for case 1 ( $\mathbf{A}-\mathbf{F}$ ) and case 2 ( $\mathbf{G}$ and H). For case 1, sagittal (A) and axial (B) MRI scans at level C4-5 on postoperative day 2 show alleviated compression without evidence of myelopathy. A T2-hyperintense signal ranging from $\mathrm{C} 2$ to $\mathrm{C} 7$ emerges on sagittal (C) and axial (D) MRI at level C4-5 performed on postoperative day 4. The 3-month follow-up sagittal (E) and axial (F) MRI at level C4-5 shows residual T2-hyperintense signal and further normalization of the spinal cord anatomy. For case 2, sagittal (G) and axial (H) MRI at level C3-4 on postoperative day 8 shows a widened spinal canal as well as a marked increase of intramedullary hyperintense T2 signal.

flavum using a Penfield dissector, after which the vertebral arches of C3-6 together with the ossified ligamentum flavum could be elevated and removed in an en bloc fashion and sent for histological evaluation. A small dural defect was closed with a direct suture and covered with hemostatic material. Additional ossified ligament was removed cranially (under the arch of $\mathrm{C} 2$ ) and caudally (under the arch of C7) using an ultrasonic aspirator (Sonopet, Stryker). Adequate decompression was achieved, and the wound was closed in multiple layers over a nonvacuum wound drain.

\section{Postoperative Course}

The histological specimen was consistent with OLF. Direct postoperative neurological evaluation demonstrated only minimally and symmetrically decreased muscle strength, which was believed to be the result of intraoperative manipulation and remained stable. On the second postoperative day, muscle strength decreased further to Medical Research Council grade 3-4 in both arms and grade 4 in both legs. Subsequent MRI showed a widened cervical spinal canal compared to the preoperative status without evidence of residual compressing structures or myelopathy on T2-weighted imaging (although expansion of the spinal cord was still limited) (Fig. 3A and $B$ ). On postoperative day 4 , the patient's muscle strength decreased even further, and she experienced an episode of severe hypotension $(40 / 20 \mathrm{~mm} \mathrm{Hg}$ ), bradycardia (30 beats per minute), and transient loss of consciousness, after which she became almost tetraplegic. Repeated MRI showed a newly emerged T2-hyperintense signal reaching from $\mathrm{C} 2$ to $\mathrm{C} 7$ (Fig. $3 \mathrm{C}$ and D). Subsequent $\mathrm{CT}$ imaging showed adequate bony decompression without complicating features (Fig. 4). After obtaining these images, we made the diagnosis of white cord syndrome, and the hypotensive/bradycardic episode was interpreted as an expression of autonomic dysregulation.

The patient was transferred to the intensive care unit, where she was treated with high-dose corticosteroids (dexamethasone $10 \mathrm{mg}$ followed by $4 \mathrm{mg}$ four times a day for 6 days) and hemodynamically supported to maintain a mean arterial pressure (MAP) of $>100$ $\mathrm{mm} \mathrm{Hg}$ for almost a week. No more episodes of autonomic dysregulation were observed. There was limited neurological recovery to a status of severe tetraparesis; she was able to slightly move the fingers on her right hand and demonstrated grade 2-3 proximal muscle strength in both legs. The patient was transferred to the 

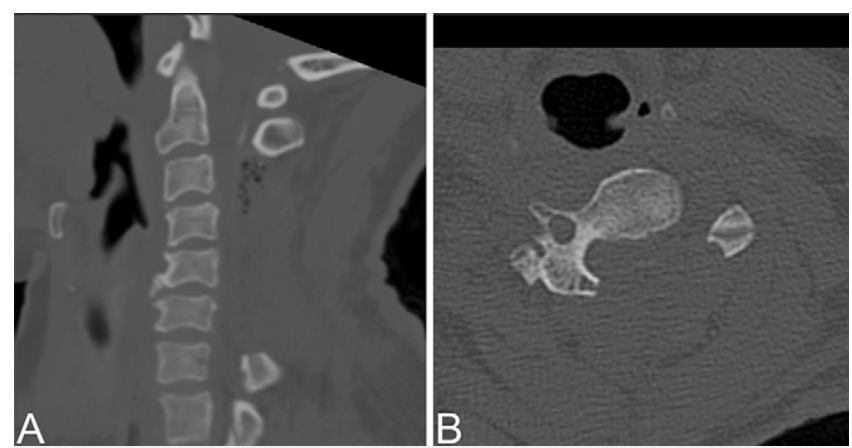

FIG. 4. Postoperative sagittal (A) and axial (B) CT scans at level C4 demonstrating adequate bony decompression without residual compressing structures.

general ward, where she stayed for 3 more weeks before being discharged to a rehabilitation center. By the time of discharge, the patient had recovered slightly more and displayed isometric proximal contractions of the right arm and grade 3-4 muscle strength of the fingers on the right hand. However, her left arm showed no movement despite grade 2 muscle strength of the fingers. Proximal and distal muscle strength in the right leg had recovered to grade 4 , whereas the left leg displayed only grade 2-3 proximal muscle strength and slight movement of the toes. The patient had persistent bowel and bladder incontinence and was discharged with a urinary catheter. Three months after discharge, she had recovered further and was able to lift her arms and stand with support. Six months after discharge, she was able to stand independently and displayed grade 4 muscle strength in all limbs.

\section{Case 2}

Presentation

A 66-year-old Dutch woman of Surinamese descent was referred to us because of walking disturbances, tingling sensations in both hands without dermatomal distribution, and deterioration of fine motor skills, which were slowly progressive over a 3-month period. Her medical history indicated hypertension. Neurological examination on admission showed normal muscle strength, a wide-based gait, a positive Lhermitte's sign, and positive Hoffmann-Trömner reflexes on both sides. Preoperative MRI showed spinal stenosis on levels C3-4 and C4-5, with an elongated T2-hyperintense signal ranging from $\mathrm{C} 2-3$ to $\mathrm{C} 6-7$, where it was most pronounced (Fig. 1E and F). CT imaging demonstrated OPLL causing spinal cord compression on levels C3-4 and C4-5 as well as a slight swan-neck deformity of the cervical spine (Fig. $1 \mathrm{G}$ and $\mathrm{H}$ ). Dynamic radiographs of the cervical spine showed some anteroposition of $\mathrm{C} 6$ relative to $\mathrm{C} 7$, which remained stable during neck flexion and extension. A C4 corpectomy with placement of an expandable cage followed by an anterior cervical discectomy and fusion of 6 -7 with anterior plate fixation was recommended to the patient.

\section{Operation}

After the patient was positioned supine in the Mayfield skull clamp with the cervical spine in slight extension, the prevertebral space from C3-4 to C6-7 was exposed. Next, a discectomy on level C6-7 was performed, the PLL was opened on both sides, and the spinal cord was locally decompressed. A partial C4 corpectomy and C3-4 and C4-5 discectomies revealed severe bony spinal cord compression caused by the ossified PLL. Bleeding of unknown origin on the right side, possibly the vertebral artery, was controlled with hemostatic material. To facilitate deformity correction and reduce the risk of future spondylosis on level C5-6, a discectomy at C5-6 was also performed. A small dural defect was covered with hemostatic material. Interbody polyetheretherketone cages (Roccia, Silony Medical) filled with autologous bone were placed in disc spaces C6-7 and C5-6, and an expandable cage (mediExpand, Medicon) was positioned in the corpectomy defect. Finally, an anterior plate (Archon, NuVasive) was placed from C3 to C7 to secure stability. Adequate spinal cord decompression and correction of the swan-neck deformity were achieved, and the wound was closed in multiple layers.

\section{Postoperative Course}

Immediate postoperative neurological examination showed no deficits, and the patient reported subjective improvement of fine motor skills in both hands. On postoperative day 1, the patient developed subtle difficulty using her left arm and hand as well as slightly decreased left-sided hand grip that remained stable over subsequent days. CT angiography performed on postoperative day 1 showed a small right-sided vertebral artery dissection flap on the level of $\mathrm{C} 4$, which was treated with a platelet antiaggregant. On postoperative day 8 , the patient's strength suddenly decreased further to near paralysis in the left arm with only some weak finger flexion, grade 4 muscle strength of the right arm, grade 3-4 muscle strength of the right leg, and grade 4 muscle strength of the left leg, which made her unable to ambulate independently. Furthermore, the patient became unable to micturate and developed bladder retention, which required placement of a urinary catheter. MRI of the cervical spine showed a widened spinal canal compared to her preoperative status as well as a marked increase of diffuse intramedullary hyperintense T2 signal congruent with white cord syndrome (Fig. 3G and H). The patient was treated with corticosteroids (dexamethasone $2 \mathrm{mg}$ two times a day) for 3 days, during which the muscle strength in her left arm partially recovered to grade 2-3, her micturition normalized, and she was able to ambulate with a walker again. Two weeks after surgery, the patient was discharged to a rehabilitation center. At the 3-month follow-up, she had improved further but was unable to walk unassisted.

\section{Discussion \\ Observations}

White cord syndrome is a rare complication of anterior or posterior cervical decompression surgery and is characterized by serious postoperative neurological deficit in the absence of apparent surgical complications, with a typical intramedullary ischemic-edematous T2-hyperintense signal on postoperative MRI. ${ }^{5}$ Five of the eight cases of white cord syndrome described in the literature emerged after posterior cervical decompression, ${ }^{7-11}$ whereas three cases occurred after anterior cervical surgery. ${ }^{5,6,12}$ We add one case description to each of those categories. The indication for decompressive surgery was cervical stenosis due to degenerative changes or herniated discs in all reported cases, except for one case in which a vertebral body tumor with epidural extension was the source of compression. ${ }^{7}$ We report the first two cases of white cord syndrome after decompressive surgery for symptomatic ossification of the cervical spinal ligaments, 
specifically PLL and LF (or yellow ligament). Although OPLL is a relatively common form of ligamentous ossification in the cervical spine, especially in East Asian populations, cervical OLF is rare, with a relative incidence of $<1 \%$ and approximately 70 reported cases in the literature. ${ }^{3,13}$ The pathophysiological mechanism underlying the transition from a normal ligament to an ossified ligament is not fully understood, but genetic factors, systemic metabolic diseases, and repetitive mechanical stress - exacerbated by abnormal spinal curvature and impaired balance-likely play a role. ${ }^{1,14}$ For cases of OLF in particular, hypoparathyroidism and the associated abnormal calcium and phosphorus levels are believed to contribute to ligamentous ossification, although an association with hyperparathyroidism also has been reported. ${ }^{15,16}$

Our first patient had a history of parathyroidectomy for primary hyperparathyroidism, which may have contributed to the development of OLF. Ossified spinal ligaments are known for their potentially dense adherence to the dura mater, and separation of the ossified ligament from the underlying dura should be performed carefully to avoid dural tears. Such adhesions were also encountered in both of our cases but especially in case 1. For this case, the Scampi technique offered an elegant method for decompressing the cervical spine posteriorly while minimizing the risk of iatrogenic central spinal cord compression. We recommend this surgical technique for future cases of symptomatic cervical OLF. An important advantage compared to standard decompression is that while removing the bone and ossifications with Kerrison punches, the risk for iatrogenic contusion in the central canal can be prevented because most compression is typically present in the midline (Fig. 1D).

The pathophysiology of white cord syndrome is believed to be reperfusion injury after sudden decompression of a chronically compressed-and possibly partly ischemic-spinal cord segment. Analogous to cerebral ischemic-reperfusion injury, disruption of the blood-spinal cord barrier and increased permeability may allow inflammatory mediators, including tumor necrosis factor-alpha, to cause oxidative stress following increased reperfusion after decompression, which leads to neuronal apoptosis. ${ }^{17,18}$ Furthermore, oxygen-free radicals within the newly available blood may damage the neuronal phospholipid membranes by lipid peroxidation (oxidative degradation), which is considered the main cause of the secondary injury-induced degenerative cascade. ${ }^{10,19}$ Lastly, direct mechanical trauma caused by acutely increased blood flow to the affected cord segment also may play a role. Although no risk factors for white cord syndrome have been identified, it is noteworthy that most case descriptions demonstrate preoperative MRI findings that suggest spinal cord edema or myelomalacia., ${ }^{5,9-11}$ These findings could imply that the duration and severity of spinal cord compression, reflected by hyperintense T2 signal on preoperative MRI, are associated with the occurrence of white cord syndrome. They may also explain the lack of MRI findings of immediate postoperative reexpansion of the spinal cord in case 1 despite the absence of residual compressing structures, because a chronically compressed spinal cord takes more time to remodel. In line with this theory, the 3-month follow-up MRI scan showed further normalization of the spinal cord anatomy.

In both of our patients, a T2-hyperintense intramedullary signal was present on preoperative MRI, although it was fairly weak in the first patient and more pronounced in the second patient. Furthermore, advanced age has been associated with a condition called "inflamm-aging," which includes a greater oxidative inflammatory response during reperfusion and possibly increases the risk of white cord syndrome. ${ }^{8}$ All but one reported case of white cord syndrome describe either an intraoperative (measured by a decrease in somatosensory and motor evoked potentials) or immediate postoperative onset of neurological deficits. The only report of late-onset white cord syndrome concerned a 79-year-old man who developed symptomatic white cord syndrome 24 hours after posterior cervical decompression and fusion. ${ }^{8}$ The authors postulated that the delayed clinical manifestation may have been related to endothelial damage and atherosclerosis caused by chronic hypertension. They suggested that a decline in nitric oxide precursors and an agedependent decrease in endothelium-dependent relaxation (which is believed to be mediated by nitric oxide) may have led to subacute reperfusion to the decompressed spinal cord area, resulting in lateonset white cord syndrome. ${ }^{20}$

We report two additional cases of delayed-onset white cord syndrome. In our first case, severe neurological deterioration started on postoperative day 2, at which point no evidence of white cord syndrome showed on postoperative MRI. When neurological deficits increased further on postoperative day 4 , an intramedullary T2-hyperintense signal emerged on MRI. In our second case, although subtle movement difficulties of the left arm were already present on postoperative day 1 , severe neurological deterioration did not manifest until postoperative day 8 , at which point MRI also showed a severe intramedullary T2-hyperintense signal. Although our patients were younger (59 and 66 years old, respectively) than the patient described by Papaioannou et al., ${ }^{8}$ both had a history of hypertension, which may have contributed to the delayed presentation in line with the previously mentioned theory.

\section{Lessons}

White cord syndrome remains a diagnosis of exclusion, and it is important to rule out all other possible causes of postoperative neurological deficit, such as iatrogenic technical trauma to the spinal cord, postoperative hematoma, incomplete decompression, malpositioned or dislocated spinal implants or grafts, and intraoperative hypotension, that could have led to spinal cord hypoperfusion. None of these conditions were present in our patients. Although class I, II, and III evidence regarding the treatment of white cord syndrome is lacking, it usually includes a combination of high-dose corticosteroids for up to 48 hours, maintaining an MAP $>85 \mathrm{~mm} \mathrm{Hg}$ for several days, and early physical therapy. ${ }^{11,21,22}$ The neurological improvement, although often partial, observed after high-dose steroids in most reported cases of white cord syndrome strengthens the hypothesis of oxidative stress and inflammatory mediators as causative factors because steroids are known to inhibit inflammation and lipid peroxidation. ${ }^{19}$ The current guidelines for blood pressure management after spinal cord injury recommend maintaining MAP at 85 to $90 \mathrm{~mm} \mathrm{Hg}$ for a duration of 5 to 7 days. ${ }^{23}$ A similar approach is mostly followed in white cord syndrome, although it is not undisputed because increasing blood flow seems counterintuitive when reperfusion injury causes the problem. ${ }^{11}$

Potent antioxidants also have been suggested to play a role in the management of spinal cord ischemic-reperfusion injury, although they are not applied in current clinical practice. ${ }^{24}$ The degree of neurological recovery varies from moderate to almost complete recovery in all reported cases. Our first patient, who was treated with high-dose corticosteroids and MAP augmentation with a minimum of $100 \mathrm{~mm} \mathrm{Hg}$ for almost a week after diagnosis, showed some recovery at discharge. Our second patient, who was treated with high-dose corticosteroids without MAP regulation (although routine measurements predominantly showed MAPs of $85-100 \mathrm{~mm} \mathrm{Hg}$ ), also showed partial recovery at 
discharge. Both patients received early physical therapy as part of standard care and were discharged to a rehabilitation center. At the 3and 6-month follow-up visits, both patients had recovered further although neurological sequelae persisted.

\section{References}

1. Ohara Y. Ossification of the ligaments in the cervical spine, including ossification of the anterior longitudinal ligament, ossification of the posterior longitudinal ligament, and ossification of the ligamentum flavum. Neurosurg Clin N Am. 2018;29(1):63-68.

2. Fujimori $T$, Watabe $T$, Iwamoto $Y$, et al. Prevalence, concomitance, and distribution of ossification of the spinal ligaments: results of whole spine CT scans in 1500 Japanese patients. Spine (Phila Pa 1976). 2016;41(21):1668-1676.

3. Dewachter L, Violon D, Crevits I. Ossification of the ligamentum flavum in the cervical spine. JBR-BTR. 2011;94(4):219.

4. Currier BL. Neurological complications of cervical spine surgery: C5 palsy and intraoperative monitoring. Spine (Phila Pa 1976). 2012; 37(5):E328-E334.

5. Chin KR, Seale J, Cumming V. "White cord syndrome" of acute tetraplegia after anterior cervical decompression and fusion for chronic spinal cord compression: a case report. Case Rep Orthop. 2013;2013:697918.

6. Giammalva GR, Maugeri R, Graziano F, et al. White cord syndrome after non-contiguous double-level anterior cervical decompression and fusion (ACDF): a "no reflow phenomenon"? Interdiscip Neurosurg. 2017;7:47-49.

7. Vinodh VP, Rajapathy SK, Sellamuthu P, Kandasamy R. White cord syndrome: a devastating complication of spinal decompression surgery. Surg Neurol Int. 2018;9(1):136.

8. Papaioannou I, Repantis T, Baikousis A, Korovessis P. Late-onset "white cord syndrome" in an elderly patient after posterior cervical decompression and fusion: a case report. Spinal Cord Ser Cases. 2019;5(1):28.

9. Antwi P, Grant R, Kuzmik G, Abbed K. "White cord syndrome" of acute hemiparesis after posterior cervical decompression and fusion for chronic cervical stenosis. World Neurosurg. 2018;113:33-36.

10. Wiginton JG IV, Brazdzionis J, Mohrdar C, et al. Spinal cord reperfusion injury: case report, review of the literature, and future treatment strategies. Cureus. 2019;11(7):e5279.

11. Mathkour M, Werner C, Riffle J, et al. Reperfusion "white cord" syndrome in cervical spondylotic myelopathy: does mean arterial pressure goal make a difference? Additional case and literature review. World Neurosurg. 2020;137:194-199.

12. Jun DS, Baik JM, Lee SK. A case report: white cord syndrome following anterior cervical discectomy and fusion: importance of prompt diagnosis and treatment. BMC Musculoskelet Disord. 2020;21(1):157.

13. Rahimizadeh A, Asgari N, Soufiani H, Rahimizadeh S. Ossification of the cervical ligamentum flavum and case report with myelopathy. Surg Neurol Int. 2018;9:263.
14. Song JY, Park JH, Roh SW. Ossified ligamentum flavum causing cervical myelopathy. Korean J Spine. 2012;9(1):24-27.

15. Sohail AH, Maan MAA, Khan MS, Masood Q. Isolated ligamentum flavum ossification in primary hypoparathyroidism. Surg Neurol Int. 2018;9(1):4.

16. Sampanis N, Gavriilaki E, Paschou E, et al. Ossification of the cervical ligamentum flavum and osseous brown tumor: late manifestations of primary hyperparathyroidism misdiagnosed in a case of parathyroid carcinoma. Clin Cases Miner Bone Metab. 2016;13(1):54-56.

17. Chen $H$, Yoshioka H, Kim GS, et al. Oxidative stress in ischemic brain damage: mechanisms of cell death and potential molecular targets for neuroprotection. Antioxid Redox Signal. 2011;14(8): 1505-1517.

18. Pan W, Banks WA, Kastin AJ. Blood-brain barrier permeability to ebiratide and TNF in acute spinal cord injury. Exp Neurol. 1997; 146(2):367-373.

19. Hall ED. Neuroprotective actions of glucocorticoid and nonglucocorticoid steroids in acute neuronal injury. Cell $\mathrm{Mol}$ Neurobiol. 1993;13(4):415-432.

20. Strolin Benedetti M, Dostert P, Marrari P, Cini M. Effect of ageing on tissue levels of amino acids involved in the nitric oxide pathway in rat brain. J Neural Transm (Vienna). 1993;94(1):21-30.

21. Bracken MB. Steroids for acute spinal cord injury. Cochrane Database Syst Rev. 2012;1(1):CD001046.

22. Hurlbert RJ. Methylprednisolone for acute spinal cord injury: an inappropriate standard of care. J Neurosurg. 2000;93(suppl 1):1-7.

23. Saadeh YS, Smith BW, Joseph JR, et al. The impact of blood pressure management after spinal cord injury: a systematic review of the literature. Neurosurg Focus. 2017;43(5):e20.

24. Shan LQ, Ma S, Qiu XC, et al. Hydroxysafflor Yellow A protects spinal cords from ischemia/reperfusion injury in rabbits. BMC Neurosci. 2010;11(1):98.

\section{Disclosures}

Dr. Arts reports grants from Zimmer-Biomet and Intrinsic Therapeutics, other from DePuy Synthes, other from Intrinsic Therapeutics, and other from NuVasive, outside the submitted work. Dr. Arts also holds a patent with DePuy Synthes and receives royalties.

\section{Author Contributions}

Conception and design: all authors. Acquisition of data: all authors. Analysis and interpretation of data: all authors. Drafting the article: Singh. Critically revising the article: Arts, de Ruiter. Reviewed submitted version of manuscript: all authors. Approved the final version of the manuscript on behalf of all authors: Singh. Study supervision: Arts, de Ruiter.

\section{Correspondence}

Ranjit D. Singh: Haaglanden Medical Center, The Hague, The Netherlands. r.singh@haaglandenmc.nl. 